\title{
Complete genome sequence analysis of a novel bunyavirus isolated from Paris polyphylla var. yunnanensis
}

\section{Zeli Chen}

Yunnan Agricultural University

\section{Rex Frimpong Anane}

Chinese Academy of Sciences

\section{Zhe Wang}

Yunnan Agricultural University

\section{Like Gao}

Yunnan Agricultural University

\section{Lu Chen}

Yunnan Agricultural University

\section{Shangyun Li}

Yunnan Agricultural University

\section{Guosong Wen}

Yunnan Agricultural University

Mingfu Zhao ( $\nabla$ 13987134765@163.com )

Yunnan Agricultural University https://orcid.org/0000-0002-1186-7203

\section{Research Article}

Keywords: YMPrNSV1, RdRp, WCLaV-2, RNA, Coguvirus

Posted Date: February 10th, 2021

DOI: https://doi.org/10.21203/rs.3.rs-209861/v1

License: (1) (1) This work is licensed under a Creative Commons Attribution 4.0 International License. Read Full License 


\section{Abstract}

A novel negative-stranded (ns) RNA virus tentatively named "Yunnan manyleaf paris rhizome negativestranded virus 1" (YMPrNSV1), was isolated from a Paris polyphylla var. yunnanensis plant exhibiting leaf chlorosis and mosaic symptoms in Yunnan. Its complete genome sequence was determined using Illumina and Sanger sequencing. The genomes composed of three RNA segments ( $L, M$ and $S$ ) with each one containing a single open reading frame. Based on sequence identity and the presence of typical bunya-like domains/motifs, the proteins encoded by YMPrNSV1 were predicted to be: RNA-dependent RNA polymerase (RdRp), putative movement protein (MP), and nucleocapsid protein (NP). Sequence comparison analyses showed that the RdRp, MP and NP of YMPrNSV1 are highly similar to those of watermelon crinkle leaf-associated virus 2 (WCLaV-2), with 69.1\%, 50.4\% and 60.9\% amino acid sequence identities respectively. Phylogenetic analysis based on deduced amino acid sequences of RdRp and NP suggested that YMPrNSV1 clustered with coguviruses in a clade, and that WCLaV-2 is the known closely related species to YMPrNSV1. Base on the above results, YMPrNSV1 should be regarded as a new member of genera Coguvirus, within the family Phenuiviridae.

\section{Introduction}

Paris polyphylla var. yunnanensis (Franch.) Hand.-Mzt., family Trilliaceae, is a perennial medicinal herb that is mainly distributed in South-west China [1,2]. The rhizome of $P$. polyphylla var. yunnanensis, known as "Chonglou" or "Dianchonglou" in China, is a famous and valuable traditional Chinese medicine (TCM). Its harvested rhizomes have become indispensable component of more than 70 popular patented medicines in China [3]. Wild P. polyphylla var. yunnanensis have been endangered as a result of excessive collection and utilization in recent years [4]. The application of artificial planting as a means to meet the market demand and to preserve wild $P$. polyphylla var. yunnanensis has contributed to the emergence of new viral diseases.

At present, four viruses have been reported to infect $P$. polyphylla var. yunnanensis, and these include the putative potexvirus Paris polyphylla virus $X$, the two potyviruses (Paris mosaic necrosis virus and Paris virus 1) and the tobamovirus Pepper mild mottle virus [5-8]. In this study, the complete genome sequence of a novel virus was identified in total RNAs extracted from diseased leaves of $P$. polyphylla var. yunnanensis. This new virus was tentatively named "Yunnan manyleaf paris rhizome negative-stranded virus 1" (YMPrNSV1).

\section{Materials And Methods}

Leaves from diseased $P$. polyphylla var. yunnanensis plant exhibiting leaf chlorosis and mosaic symptoms were collected from a field in Yunnan, China (Fig. 1A). Total RNA was isolated from diseased plant leaves using Plant RNA Kit (Omega Biotek, USA). High-throughput sequencing (HTS) was performed on the Illumina HiSeqTM 2500 platform (OE biotech. Shanghai, China), followed by processing of the raw reads into clean reads and de novo assembly into contigs [9]. The viral contigs were obtained by NCBI 
BLAST analysis of the assembled contigs. ORF prediction and identification of domains in the putative proteins were performed using the NCBI ORF Finder (https://www.ncbi.nlm.nih.gov/orffinder/) and Conserved Domain Database (https://www.ncbi.nlm.nih.gov/Structure/cdd/wrpsb.cgi) search tools. Modeling prediction and analysis of putative proteins were performed with the Phyre2 web portal [10]. Comparison of nucleotide and amino acid sequences were conducted by BioEdit 7.0 using ClustalW multiple alignments tool. Phylogenetic analysis was performed in MEGA 7.0 using the maximumlikelihood (ML) algorithm with 1000 bootstrap replicates.

To verify the presence of viruses in symptomatic samples, we used reverse transcription PCR (RT-PCR) and specific primers (Table S1) designed from the sequences of the assembled viral contigs. RT-PCR was performed using Prime Script ${ }^{\mathrm{TM}}$ II 1st Strand cDNA Synthesis Kit (TaKaRa Bioengineering, Dalian) and 2xTaq PCR Master Mix Kit (BioTeke, Beijing) on diseased leaf samples used for HTS. The sequences of the extreme ends of the genomic RNAs were determined by employing the 5'- and 3'-RACE kits (BioTeke, Beijing) following the manufacturer's instructions. Amplicons were gel-purified, cloned and sequenced by Sanger Sequencing (Sangon biotech. Shanghai, China) according to standards protocols [11].

\section{Results}

De novo assembly of the $25,057,448$ clean reads obtained through HTS of total RNAs purified from symptomatic leaves of $P$. polyphylla var. yunnanensis plant, generated a total of 111,275 contigs. These contigs were then subjected to BLASTn and BLASTx search against nucleotide and amino acid (aa) sequences of the NCBI databases, to obtain the virus contigs. Twelve contigs encoding putative proteins with high amino acids (aa) sequence identity (52.11-69.76\%) with the RNA-dependent RNA polymerases (RdRp), the nucleoprotein (NP) and the putative movement protein (MP) of watermelon crinkle leafassociated virus 2 (WCLaV-2), a novel plant-infecting bunyavirales-related virus reported recently [12], were identified. These results suggest that a potential new bunyavirus may be responsible for the viruslike infection observed in diseased plants.

The complete genome of YMPrNSV1 consist of three RNAs (Fig. 1B), with a total size of 9,506 nt (GenBank accession numbers MT269667-MT269669). RNA1 is the largest (L) segment and encodes one putative ORF of 2,181 amino acids. BLASTp analysis showed that the $L$ protein has RdRp structure that is typical of members of the family Phenuiviridae, order Bunyavirales [13], and RdRp of WCLaV-2 had the highest aa sequence identity of $69.82 \%$ with that of YMPrNSV1 (Table S2). Additionally, the conserved Bunya_RdRp superfamily domain (cl20265; E-value: 2.92e-40) of members of the order Bunyavirales was identified in the L protein of YMPrNSV1 (Table S2). Pairwise comparisons of the $L$ protein sequence of YMPrNSV1 with those of other nsRNA viruses indicated that, YMPrNSV1 contains the typical six motifs (premotif A and motifs A-E) that are highly conserved in the RdRps of members of this taxon. WCLaV-2, watermelon crinkle leaf-associated virus 1 (WCLaV-1), citrus leaf flecking-associated virus (CLFaV), and members of the genus Coguvirus shared the highest sequence identity with YMPrNSV1 (Table 1, Fig. 1C). YMPrNSV1 L protein contains the motif A $\left({ }^{942}\right.$ DATKWC $\left.{ }^{947}\right)$, motif B $\left({ }^{1035}\right.$ QGILHYTSS $\left.{ }^{1043}\right)$, motif C $\left({ }^{1076} \mathrm{SDD}^{1078}\right)$, and motif D with the ${ }^{1114} \mathrm{YXKX}_{2} \mathrm{KS}^{1120}$ sequence (Fig. 1C). The motif E tetrapeptide 
${ }^{1129} \mathrm{EYXS}^{1132}$ (Fig. 1C) which is highly conserved in the polymerases of segmented nsRNA viruses [14, 15].

Three basic amino acid residues $(K, R$, and $R / K)$ and their downstream residues containing glutamic acid $(E)$ that are present in premotif $A$ and conserved in bunyavirus $R d R p s[16,17]$ were identified in YMPrNSV1. The conserved endonuclease motif $\mathrm{H}_{66} \mathrm{D}_{78} \mathrm{PD}_{95-96} \mathrm{ExG}_{107-109} \mathrm{~K}_{126}$ involved in capping, a strategy used by negative-stranded viruses to translate viral proteins by using capped terminal ends from their host mRNAs [18], was located in the N-terminal region of YMPrNSV1 L protein. A variation ExG was observed in the ExT/A domain, which conserved in RdRp of most bunyaviruses [18]. Altogether, these results indicate that the L protein encoded by RNA1 of YMPrNSV1 is a bunyaviral RdRp. In addition, the core RdRp domain of YMPrNSV1 is closely related to those of WCLaV-2, WCLaV-1 and other coguviruses, and shares $81.3 \%$ aa sequence identity with WCLaV-2 (Table 1 ).

Tabe 1 Amino acid sequences identities (\%) between YMPrNSV1 and other (-ss) RNA viruses. 


\begin{tabular}{|c|c|c|c|c|c|c|}
\hline Genus & Virus & $\mathrm{RdRp}$ & Core RdRp & MP & NP & Core NP \\
\hline \multirow[t]{9}{*}{ Coguvirus } & CCGaV & 59.5 & 72.6 & 42.8 & 41.5 & 55 \\
\hline & CiVA & 59.6 & 74.8 & 40.7 & 38.9 & 46.5 \\
\hline & GaCLV-1 & 37.7 & 46.5 & 18.8 & 27.6 & 29.2 \\
\hline & GaCLV-2 & 27.3 & 44.6 & 14.2 & 19 & 22.8 \\
\hline & GaCLV-3 & 27.5 & 43.8 & 14.3 & 18.4 & 22.5 \\
\hline & GaCLV-4 & 29.4 & 44.1 & 14.8 & 24.5 & 28.1 \\
\hline & WCLaV-1 & 60.4 & 75.4 & 39.3 & 48.4 & 54.5 \\
\hline & WCLaV-2 & $69.1^{*}$ & 81.3 & 50.4 & 60.9 & 62.0 \\
\hline & CLFaV & 59.7 & 72.9 & 42.5 & 41.5 & 52.1 \\
\hline \multirow[t]{2}{*}{ Bandavirus } & SFTSV & 15.2 & 23.7 & - & 9.4 & 12.9 \\
\hline & BHAV & 15.4 & 24.4 & - & 13.2 & 17 \\
\hline Laulavirus & LLV & 26.5 & 44.1 & - & 17.3 & 20.5 \\
\hline Lentinuvirus & LeNSRV2 & 24.4 & 34.3 & - & 16.4 & 14.2 \\
\hline Entovirus & EnPLV1 & 22.4 & 33.6 & 14.1 & 17.3 & 17.4 \\
\hline Phasivirus & PCLV & 14 & 21.3 & - & 12.1 & 14.2 \\
\hline \multirow[t]{3}{*}{ Phlebovirus } & HYSV & 15.2 & 23.5 & - & 9.4 & 12.9 \\
\hline & RVFV & 14.6 & 22.7 & - & 9.4 & 12.5 \\
\hline & PuTV & 15.6 & 24.5 & - & 10.6 & 14.6 \\
\hline \multirow[t]{2}{*}{ Rubodvirus } & ARWV2 & 16.4 & 25.9 & 8.3 & 13.8 & 19.3 \\
\hline & ARWV1 & 15.8 & 25.1 & 7.9 & 13.7 & 20.4 \\
\hline \multirow[t]{2}{*}{ Tenuivirus } & RSV & 12.2 & 24.6 & 8.1 & 7.7 & 10.9 \\
\hline & RHBV & 12.7 & 24.6 & 7.6 & 6.2 & 9.8 \\
\hline \multirow[t]{3}{*}{ Uukuvirus } & UUKV & 15.8 & 22.9 & - & 13.7 & 18.4 \\
\hline & SILV & 16.5 & 23.2 & - & 10.8 & 14.2 \\
\hline & KASDV & 15.8 & 23.5 & - & 9.1 & 12.1 \\
\hline \multirow[t]{3}{*}{ Orthotospovirus } & TSWV & 7.6 & 8.3 & - & 7 & 10.4 \\
\hline & $\mathrm{CaCV}$ & 7.2 & 8.4 & - & 6.9 & 9.1 \\
\hline & WSMoV & 7.3 & 8.3 & - & 7.2 & 9.5 \\
\hline
\end{tabular}




\begin{tabular}{|lllllll|}
\hline Genus & Virus & RdRp & Core RdRp & MP & NP & Core NP \\
\hline Orthonairovirus & DUGV & 5.8 & 7.3 & - & 5.7 & 4.9 \\
\hline Emaravirus & EMARaV & $7.0^{* *}$ & 9.4 & - & 5.6 & - \\
& RRV & 7.3 & 9.1 & - & 4.5 & - \\
\hline Herbevirus & HEBV & 8.1 & 7 & - & 5.4 & 3.6 \\
\hline Feravirus & FERV & 7.7 & 6.2 & - & 5.8 & - \\
\hline Jonvirus & JONV & 7.9 & 7.6 & - & 4.9 & - \\
\hline *Numbers in red represent highest identities of YMPrNSV1 with selected (-ss) RNA viruses. \\
\hline \multirow{2}{*}{ **Numbers in blue represent lowest identities of YMPrNSV1 with selected (-ss) RNA viruses. } \\
\hline
\end{tabular}

RNA2 is the medium (M) segment, encoded by one ORF, with a molecular mass of $46.2 \mathrm{KDa}$ (Fig. 1A). The proteins most similar to the deduced aa sequences of RNA2 identified by BLASTp analysis were the putative MP of WCLaV-2 and viruses of the genus Coguvirus (CiVA and CCGaV), with aa pairwise identity ranging from 40.7 to $50.4 \%$ (Table 1, table S2). Therefore, the deduced $46.2 \mathrm{KDa}$ protein of YMPrNSV1 may be the putative MP. Nevertheless, no significant conserved domains in MP of YMPrNSV1 were identified using CDD analyses (Table S2).

RNA3 is the small (S) segment, contained an ORF coding for putative protein of 345 aa, with a molecular mass of $38.68 \mathrm{kDa}$ (Fig. 1A). BLASTp search identified NP of WCLaV-2 as the protein that shared the highest identity with the putative $38.68 \mathrm{kDa}$ protein encoded by RNA3 of YMPrNSV1 (Table S2).

According to the CDD analyses, RNA3 encodes proteins that contain the conserved Tenui_N super family domains (accession cl05345), the typical domains in NP of viruses in the family Phenuiviridae, with a Evalue of $9.85 \mathrm{e}-15$ (Table S2). In agreement with this finding, when analysed with Phyre2 [10], the NP protein of Rift Valley fever virus (RVFV; genus Phlebovirus) was found to be the best template for modeling (with $100 \%$ confidence) the tertiary structure of the putative $38.68 \mathrm{kDa}$ protein region between positions 70 to 274 (about $59 \%$ of the full-length protein). Based on these results, the $38.68 \mathrm{kDa}$ protein is very likely to be the NP of YMPrNSV1. Pairwise comparison analysis showed that the aa sequence identities between the NP of YMPrNSV1 and other nsRNA viruses of the family Phenuiviridae and order Bunyavirales, ranged from 4.5 to $60.9 \%$. The core NP of WCLaV-2, WCLaV- 1 and coguviruses presented the highest aa identities with YMPrNSV1 (Table 1).

To confirm the taxonomic classification of YMPrNSV1, phylogenetic trees inferred from RdRp and NP amino acid sequences of YMPrNSV1 and other nsRNA bunyavirales were constructed. According to the generated trees, YMPrNSV1 clustered with coguviruses (Fig. 2 and Fig. S1). Most members of the coguvirus clade, including three tentative members (WCLaV-1, WCLaV-2, and citrus leaf fleckingassociated virus) are known infecting plants. These results are consistent with similar phylogenetic trees constructed elsewhere $[19,20]$. WCLaV-2, recently reported to infect watermelon in Henan Province, China 
[12], is the closest known related to YMPrNSV1 with $100 \%$ and $84 \%$ bootstrap support (based on the RdRp and NP aa sequences respectively) (Fig. 1 and Fig. S1). According to the criteria proposed by ICTV Phenuiviridae Study Group, any virus having $<95 \%$ RdRp encoded by RNA1 aa sequence identity to the closest protein homolog (https://talk.ictvonline.org/) should be considered as a new species in the genus Coguvirus. In this study, the RdRp aa sequence identity value of YMPrNSV1 and WCLaV-2 is $69.1 \%$, a value below the threshold for a new species.

\section{Discussion}

In the present study, we discovered and characterized a novel plant-infecting nsRNA viruses with the tentative named "Yunnan manyleaf paris rhizome negative-stranded virus 1" (YMPrNSV1) using HTS and Sanger sequencing. YMPrNSV1 was isolated from P. polyphylla var. yunnanensis, and its complete genome shares many features that are common of viruses in the order Bunyavirales. The $L$ and $S$ segments have a negative polarity, encoding RNA-dependent RNA polymerase and nucleocapsid (N) proteins respectively. The $\mathrm{M}$ segment codes for the putative movement protein (MP) in a positive-sense genomic organization. The conserved sequences SDD in motif $C$, the signature of the core domain in RdRp for segmented negative-stranded RNA viruses in the families Orthomyxoviridae, Arenaviridae, and the previously Peribunyaviridae [12], was also identified in the RdRp of YMPrNSV1. Likewise, the sequence $E Y X S$ in motif $E$, which is typically conserved in the polymerases of segmented nsRNA viruses $[21,18]$ was also identified, indicating that YMPrNSV1 is a segmented nsRNA virus. Sequence and phylogenetic analyses showed that YMPrNSV1 was closely related to WCLaV-2, with $69.1 \%$ identity in the RdRp aa sequence, and also clustered with WCLaV-2 in a unique branch in the coguvirus group. Base on the above results, YMPrNSV1 should be regarded as a new member of genera Coguvirus, which is a new genus in the family Phenuiviridae, order Bunyavirales [22].

\section{Declarations}

\section{Compliance with ethical standards}

\section{Funding}

This study was funded by the National Natural Science Foundation of China (81860774).

\section{Conflict of interest}

The authors declare that they have no conflict of interest.

\section{Ethical approval}

This article does not contain any studies with human participants or animals performed by any of the authors.

\section{Data availability}


The dataset generated during the current study is available in the GenBank database (accession no. MT269667- MT269669).

\section{References}

[1] Li B, Ruotti V, Stewart RM et al (2009) RNA-Seq gene expression estimation with read mapping uncertainty. Bioinformatics 26:493-500.

[2] Chase MW, Christenhusz M, Fay M et al (2016) An update of the Angiosperm Phylogeny Group classification for the orders and families of flowering plants:APG IV. Bot J Linn Soc 181: 1-20.

[3] Qin XJ, Ni W, Chen CX et al (2018) Seeing the light: shifting from wild rhizomes to extraction of active ingredients from above-ground parts of Paris polyphylla var. yunnanensis. J Ethnopharmacol 224:134139.

[4] Huang Y, Zhou N, Yang M, et al (2019) Comparative study of the population genetics of wild and cultivated populations of Paris polyphylla var. yunnanensis based on amplified fragment length polymorphism markers. Ecol Evol 9:10707-10722.

[5] Dong JH, Ding M, Fang Q et al (2007) Molecular identification of a Potexvirus isolate infecting Pairs polyphylla var. Yunnanensis and analysis of 3'terminal sequence. Acta Phytopathologica Sin 37(3):237241

[6] Lan P, Zhao J, Zhou Y et al (2018) Complete genome sequence of Paris mosaic necrosis virus, a distinct member of the genus Potyvirus. Arch Virol 163(3):78

[7] Chen L, Anane R F, Wang Z et al (2020) Whole-genome sequence analysis of paris virus 1: a novel member of the genus Potyvirus infecting Paris polyphylla var. yunnanensis. Arch Virol 165:985-988

[8] Wen GS, Yang LY, Anane RF, Chen ZL et al (2019) First report of Pepper mild mottle virus in Pairs polyphylla var. Yunnanensis in China. Plant Dis 103:3289

[9] Cao MJ, Zhang S, Li M et al (2019) Discovery of Four Novel Viruses Associated with Flower Yellowing Disease of Green Sichuan Pepper (Zanthoxylum armatum) by Virome Analysis. Viruses 11:696.

[10] Kelley LA, Mezulis S, Yates CM et al (2015) The Phyre2 web portal for protein modelling, prediction and analysis. Nat Protoc 10:845-858.

[11] Navarro B, Minutolo M, De Stradis A et al (2018). The first phlebo-like virus infecting plants: a case study on the adaptation of negative-stranded RNA viruses to new hosts. Mol Plant Pathol 19:1075-1089.

[12] Xin M, Cao M, Liu W et al (2017) Two negative-strand RNA viruses identified in watermelon represent a novel clade in the order Bunyavirales. Front Microbiol. 8:1514. 
[13] Navarro B, Minutolo M, De Stradis A et al (2018) The first phlebo-like virus infecting plants: a case study on the adaptation of negative-stranded RNA viruses to new hosts. Mol Plant Pathol 19:1075-1089.

[14] van Poelwijk F, Prins M, and Goldbach R (1997) Completion of the impatiens necrotic spot virus genome sequence and genetic comparison of the $L$ proteins within the family Bunyaviridae. J Gen Virol 78:543-546

[15] Kormelink R, Garcia ML, Goodin M et al (2011) Negative-strand RNA viruses: the plant-infecting counterparts. Virus Res 162:184-202.

[16] Bruenn J A (2003) A structural and primary sequence comparison of the viral RNA dependent RNA polymerases. Nucleic Acids Res 31:1821-1829.

[17] Elbeaino T, Digiaro M, Alabdullah A et al (2009). A multipartite single-stranded negative-sense RNA virus is the putative agent of fig mosaic disease. J Gen Virol 90:1281-1288.

[18] Reguera J, Weber F and Cusack S (2010) Bunyaviridae RNA Polymerases (L-Protein) Have an NTerminal,Influenza-Like Endonuclease Domain, Essential for Viral Cap-Dependent Transcription. PLOS Pathog 6:e1001101.

[19] Diaz-Lara A, Navarro B, Di Serio F et al (2019) Two Novel Negative-Sense RNA Viruses Infecting Grapevine Are Members of a Newly Proposed Genus within the Family Phenuiviridae. Viruses 11:685.

[20] Navarro B, Zicca S, Minutolo M et al (2018) A Negative-Stranded RNA Virus Infecting Citrus Trees: The Second Member of a New Genus Within the Order Bunyavirales. Virology 9.

[21] Müller R, Poch O, Delarue M (1994) Rift valley fever virus L segment: correction of the sequence and possible functional role of newly identified regions conserved in RNA-dependent polymerases. J Gen Virol 75:1345-1352.

[22] Kuhn J H, Adkins S, Alioto D et al (2020) taxonomic update for phylum Negarnaviricota (Riboviria: Orthornavirae), including the large orders Bunyavirales and Mononegavirales. Arch Virol 165:3023-3072.

\section{Figures}


A

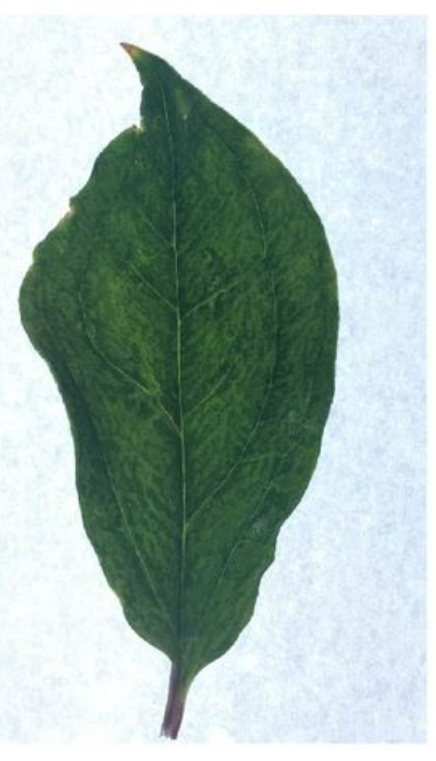

B

RNA1

$(6,616 \mathrm{nt})$

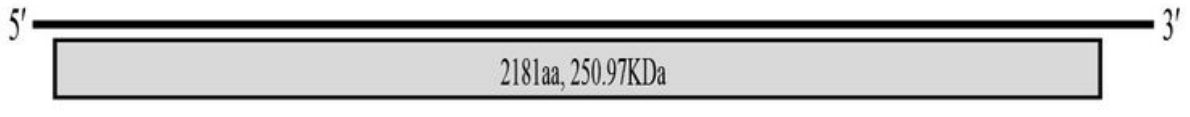

6,564

RNA dependent RNA polymerase

RNA2

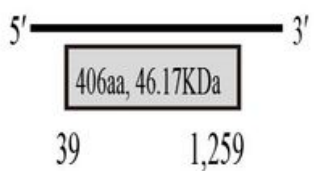

putative movement protein

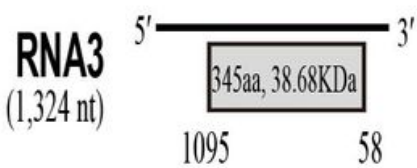

nucleocapsid protein

C

Premotif A

Motif A

Motif B

Motif C

Motif D

Motif E

WCLaV-1

WCLaV-2

RVFV

RSV

TSWV

CLFaV

CCGaV

CiVA

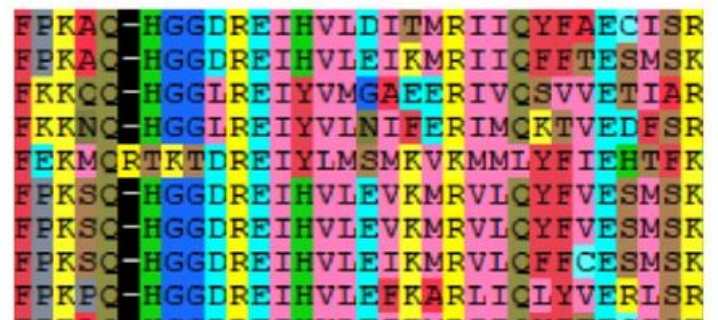

SADATKWCQRND
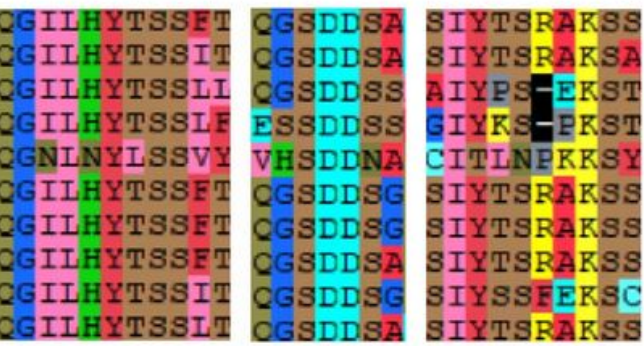

EYNSE

SADATKWCCRND

DDARKWNCGH

KRSRIAFLSADC

SADATKWCCRND

SADATKWCQRND

SADATKWCQRND

YMPrNSV1 EPKAd-HGGDREIHVLEIKMRIICYETECISK

SADATKWCCRND

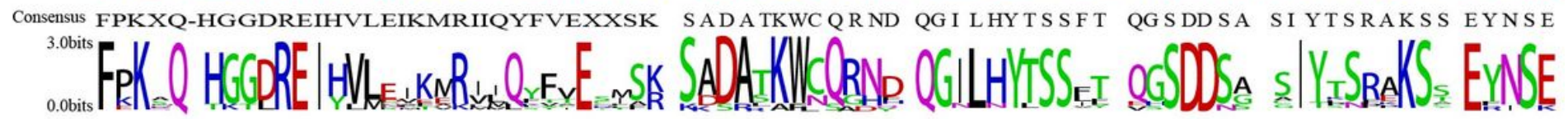

\section{Figure 1}

(A) Symptomatic leaf of P. polyphylla var. yunnanensis. (B) Coding strategy and genome segments of YMPrNSV1. Open reading frames (ORFs) and deduced products of each RNA are shown as gray boxes, with the amino acid (aa) length, estimated molecular weight $(\mathrm{kDa})$, and function of the putative proteins. (C) Multiple alignment of RdRp conserved motifs (premotif A and motifs A-E) present in YMPrNSV1 and other negative-stranded RNA viruses. 


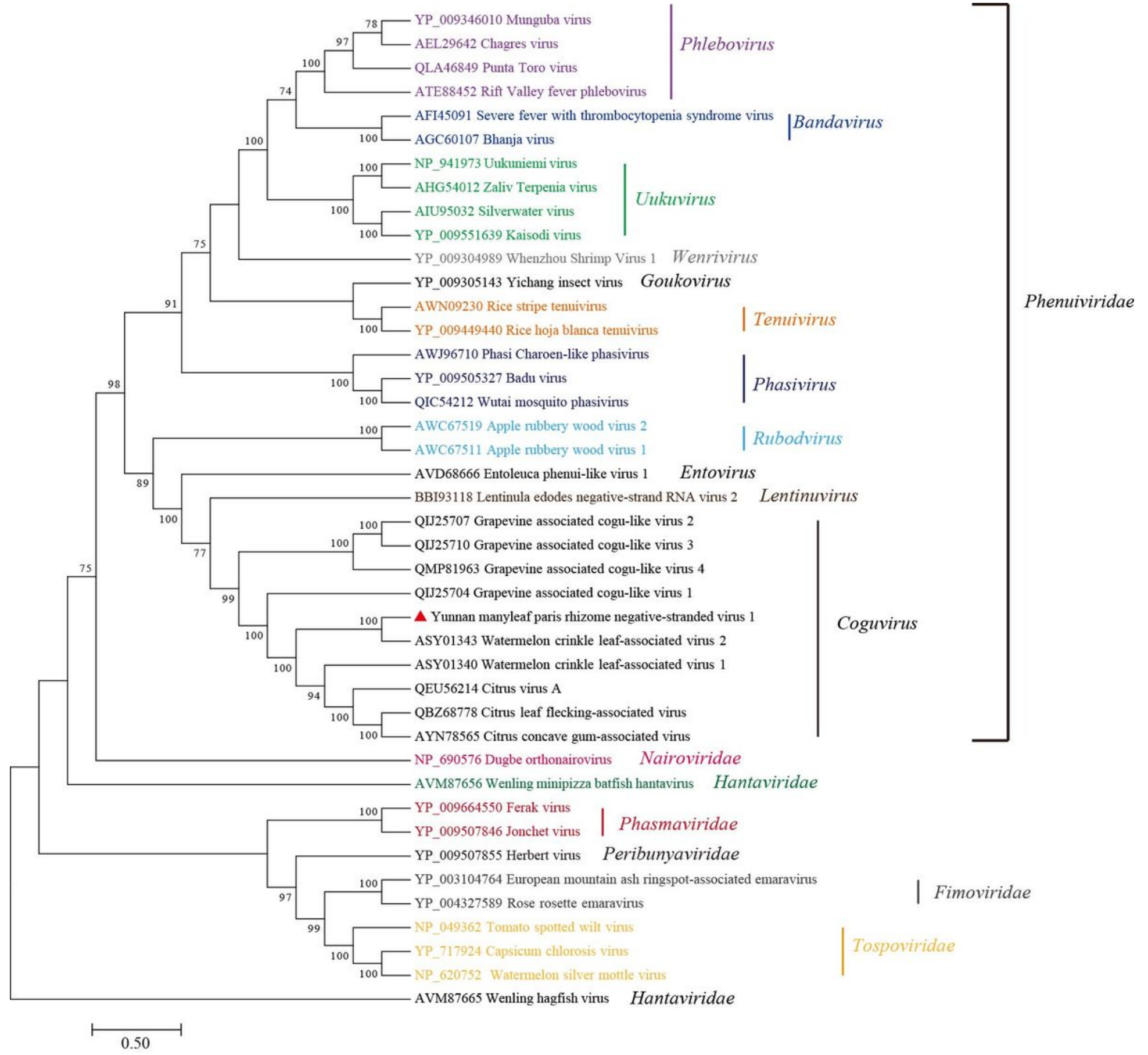

\section{Figure 2}

Maximum likelihood (ML) phylogenetic tree of YMPrNSV1 and representative negative-sense RNA viruses in the order Bunyavirales based on the amino acid sequence of RdRp. Bootstrap probabilities for each branch node were estimated using 1,000 replicates and those above 50\% are shown. The scale bar represents number of substitutions per amino acid site. Viruses in the same genera or family are labeled with the same colors. YMPrNSV1 was marked with red triangle.

\section{Supplementary Files}


This is a list of supplementary files associated with this preprint. Click to download.

- Supplementarymaterials.doc

- ThecompletegenomesequenceofYMPrNSV1.pdf 